This item was submitted to Loughborough's Research Repository by the author.

Items in Figshare are protected by copyright, with all rights reserved, unless otherwise indicated.

Incorporating landmarks in driver navigation system design: an overview of results from the REGIONAL project

PLEASE CITE THE PUBLISHED VERSION

PUBLISHER

(c) Royal Institute of Navigation

LICENCE

CC BY-NC-ND 4.0

REPOSITORY RECORD

May, Andrew, Tracy Ross, and Steven H. Bayer. 2019. "Incorporating Landmarks in Driver Navigation System Design: An Overview of Results from the REGIONAL Project”. figshare. https://hdl.handle.net/2134/844. 


\title{
Incorporating Landmarks in Driver Navigation System Design: An Overview of Results from the REGIONAL Project
}

\author{
Andrew J. May, Tracy Ross and Steven H. Bayer \\ (Loughborough University, UK) \\ (Email: A.J.May@1boro.ac.uk)
}

This paper presents an overview of results from the 2 year REGIONAL project. The aims of REGIONAL were to undertake research to enable landmarks to be an integral feature of future vehicle navigation systems. Results from the project, including 5 empirical road-based trials, are summarised. The main findings were: landmarks were widely used by drivers as key navigation cues; the incorporation of good landmarks within navigation instructions has the potential to considerably enhance vehicle navigation systems; although a wide range of landmarks are potentially useful to a driver, only a limited set, which displayed key characteristics, were consistently effective as navigation cues.

\section{KEY WORDS}

1. Navigation. $\quad$ 2. Vehicle. $\quad 3$. Design.

\section{INTRODUCTION.}

1.1. Background. This paper provides research findings that can be used to potentially enhance the design of future driver navigation aids (e.g. 'navigation', 'satellite navigation' or 'route guidance' systems). It is recognised that as well as being a common activity, navigating in unfamiliar road environments is a demanding cognitive task for drivers (Burnett, 1998). It has long been established that drivers have difficulties in planning and following efficient routes to destinations (King, 1986; Streeter, 1986; Wierwille, 1993), with resulting stress, delays and potentially unsafe road behaviour such as late lane changes or attempting to read paper maps while driving. Vehicle navigation systems provide a technological solution to planning and following routes usefully by presenting in-vehicle navigation instructions to drivers derived from navigable map databases. Current systems make very little use of landmarks as key navigation cues - they tend to present a combination of map views and/or turn-by-turn navigation information, and still rely on distance-to-turn information and junction layout representations to identify a forthcoming manoeuvre to a driver. 


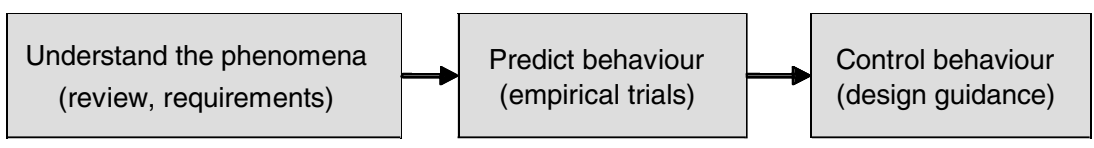

Figure 1. The REGIONAL project approach.

Authors such as Burnett (1998) have argued that navigation systems should be more naturalistic, i.e. their behaviour should approximate a passenger with detailed route knowledge providing context-based navigation instructions to the driver as required. A key characteristic of these types of instructions is the use of landmarks as navigation cues, for example: 'turn right after the petrol station', 'go straight over the traffic lights' and 'keep going past the train station'. There are strong theoretical arguments that support the contention that landmarks should be an integral part of future vehicle navigation systems:

(1) they are consistent with basic human navigation strategies as they form key elements within cognitive maps of the environment, aid the learning of the environment (Golledge, 1993; Evans, 1984), and are used in way-finding strategies (Alm, 1990);

(2) they are valued by drivers when navigating (Burns, 1997; Streeter, 1986; Burnett, 1998; Wochinger \& Boehm-Davis, 1997); and

(3) they can improve the usability of vehicle navigation systems by potentially increasing driver confidence and satisfaction, and decreasing navigation errors (Bengler, Haller, \& Zimmer, 1994; Burnett, 1998; Alm, Nilsson, Jarmark, Savelid, \& Hennings, 1992; Green, Hoekstra, Williams, Wen, \& George, 1993).

1.2. Aim. The specific aim of this paper is to provide a summary of results arising from the REGIONAL Project. REGIONAL was an academic/commercial research collaboration, funded by the UK government via the EPSRC under the LINK Inland Surface Transport programme, and running from 1999 to 2002.

2. PROJECT OVERVIEW. The overall aims of REGIONAL were simple: to identify and develop the knowledge necessary to ensure that landmarks can become an integral part of future vehicle navigation systems. The approach taken within the project was an inductive/deductive approach as shown in (Figure 1).

The bulk of the research consisted of a range of empirical studies that addressed key research questions relating to either 'choosing', 'using' or 'presenting' landmarks within future vehicle navigation systems. The main findings from a series of research activities are presented in the following sections: 3. Research review; 4. Industry consultation; 5. Requirements study: Drivers' use of landmarks; 6. Requirements study: Drivers' use of navigation information; 7. Statistical modelling: landmark 'effectiveness'; 8. Road trial: Driver performance with landmarks and distance information; 9. Road trial: Navigation using traffic lights; 10. Road trial: Impact of information reliability; the Conclusions are presented in section 11. The reader is welcome to contact the authors for more details of any of this work. 


\section{RESEARCH REVIEW.}

3.1. Aims and method. The aims of the research review were to understand the 'state-of-the-art' knowledge relating to key research questions including the following: (1) What is a landmark? (2) How can the driver's navigation task be defined? (3) What landmarks are potentially suitable for supporting the navigation task? (4) What factors might influence the effectiveness of landmarks for navigation? (5) How might landmarks be presented to the driver? (6) What are appropriate methods and measures for investigation the use of landmarks by vehicle navigation systems?

Relevant research literature was identified from a diverse range of academic disciplines; the main findings are outlined below.

3.2. Summary of main findings. Following the approach taken in much of the literature, the REGIONAL working definition of a 'landmark' included buildings, street furniture and built aspects of the environment (e.g. bridges), but excluded geographical features such as hills and bends in the road, and also direction signs and street name signs (as these are information cues used on the basis of their information content rather than their visual appearance as an object).

Various theoretical perspectives on driver navigation were reviewed. A navigation model proposed by Burnett, (1998) was used as a basis for defining the navigation task. This model focuses on the temporal nature of the information requirements, and the need for information to preview navigation decision points, identify the geographical location of manoeuvres, and maintain driver confidence.

In addition, a range of studies was identified which had specifically investigated landmarks in the context of vehicular navigation. These tended to either be requirements studies which listed the most frequently used landmarks (invariably traffic lights, traffic signs, shops, petrol stations and bridges) within the context of a particular study, or laboratory-based trials of a simulated HMI that incorporated landmarks. Within a driving context, there was often little attempt to understand the basic characteristics of landmarks, and their context, that determine their navigational effectiveness.

Finally, it was clear that there was a lack of design advice (especially in terms of presenting landmarks) which could be used directly by systems manufacturers to enhance their products. A notable exception was the work by Pauzie, (1997) that recommended that specific labels are better for well-known landmarks (e.g. 'McDonalds ${ }^{\mathrm{TM}}$, rather than 'fast food restaurant'), but for less 'branded' objects (e.g. churches) a generic description relating to its form or function is preferable.

\section{INDUSTRY CONSULTATION.}

4.1. Aims and method. A consultation exercise was carried out with key stakeholders within industry who would influence the design and manufacture of future vehicle navigation aids. This ongoing industry consultation exercise helped ensure that the outputs of the REGIONAL project were relevant to commercial systems developers. It was therefore necessary to understand:

- The current process involved in navigation system design, including the roles of different stakeholders (e.g. navigable map database providers, navigation systems developers and automotive manufacturers). 
- The technology that underpins the functioning of current and emerging navigation systems.

- The constraints that are current and potential barriers to designing more effective vehicle navigation systems.

- Relevant technological and market trends

As well as using information gained from the research review (above), the main method employed during the industry consultation phase of the project was a series of semi-structured interviews with a range of personnel from the stakeholders mentioned above.

4.2. Summary of main findings. A summary of some key points is given below: For database development:

- A strong business case is essential for landmarks to be included in future navigable map databases. The business case for collecting and maintaining the necessary data relies on there being multiple uses for this data.

- The source data must be available, accessible, accurate and easily maintainable; some landmarks already exist on navigable databases as Points of Interest (POIs).

- The selection of landmarks should, as far as possible, not rely on field visits to verify their existence and location.

- Once obtained, landmark data should be easily maintained by (a) selecting landmarks which are unchanging or (b) selecting those where updates will be automatically notified.

For navigation system software development:

- Guidance must be given on the 'rules' for use of landmarks, whether these are generic (e.g. 'where a landmark of type [xxxx] exists on the database, use it') or more specific (e.g. "if there are more than 3 turnings within $100 \mathrm{~m}$, and the landmark is on the same side as the turnings and within $10 \mathrm{~m}$ of the target turning, present the landmark').

- For each potential usage scenario, the 'proof' is needed that any particular approach (set of rules) is the optimum within the operational constraints at the time.

- Landmarks must be considered within the context of a wider set of information that is available to the driver (e.g. distance and road layout information, local and national signage).

For Human-Machine Interface (HMI) design:

- The use and design of landmark information must enhance, not reduce, current customer perception of reliability, value and trust in the navigation system.

- Guidance must be given on the implementation of landmarks within the HMI, e.g.: whether the information should complement or replace that currently provided; whether information presentation should be verbal, visual or both; whether icons/descriptions should be generic or specific.

- The use and design of landmark information should not constrain the internationalisation of products. 
5. REQUIREMENTS STUDY: DRIVERS' USE OF LANDMARKS.

5.1. Aims. The main aims of this study were to begin to understand the phenomena (Figure 1): to establish which landmarks were typically required for driver navigation and to identify the salient characteristics of these landmarks.

5.2. Method. Three linked routes were identified within a town (population approx. 60,000) environment. These routes covered a total of approximately $16 \mathrm{~km}$, involved 19 distinct decision points (i.e. junctions where navigational uncertainty would be expected), and took approximately 25 minutes to drive. 32 participants (16 male, 16 female; age range 22 to 60 ; all experienced drivers) were asked to write down the information they felt an unfamiliar traveller would need to drive and navigate these three linked routes successfully. An experimental design was used such that the information identified was based on cues which were highly visually prominent, and those which registered prominently in individuals' cognitive maps of the area. Therefore, half of the participants had no prior experience of the area and wrote down their navigation instructions based on observing video footage of the routes (video group). They were permitted to rewind and review any part of the video until they were happy with their route descriptions. The other half of the participants had detailed knowledge of the geographical area (having lived and/or worked in the area for at least five years) and wrote down their navigation instructions based on recall from memory. This group was provided with bare route schematics that identified the desired route, but had all information such as street names, junction types, geographical features and potential landmarks removed. All participants were informed that the study was investigating the directions people use for navigation; to avoid biasing the instructions they provided, no mention was made of any specific types of information cues, including landmarks.

Having generated navigation directions for all three routes, all landmarks used by that participant were highlighted within the written instructions. A semi-structured interview technique was then used to identify the reasons for the inclusion of particular landmarks within the navigation directions (e.g. why that particular landmark was used, what made it stand out from other potential information etc).

5.3. Results. A detailed description of the route was developed, including all potential landmarks that were en-route. Data was then coded to identify the frequency of use of particular landmark categories (e.g. traffic lights). Each particular landmark within that category was assigned a numbered code (e.g. traffic lights were individually identified as TL1, TL2 etc). Sainburys ${ }^{\mathrm{TM}}$ supermarket was individually coded as it was the only large supermarket on the route. MacDonalds ${ }^{\mathrm{TM}}$ restaurant was individually coded because it was much more visually distinctive than other cafes or restaurants en-route.

In addition to those landmarks that were identified by participants, the total number of occurrences of that type of object on the route was recorded, see Table 1. Example: there were 2 bridges en-route, both of these were referred to by participants, with a total of 26 references to bridges by the 32 participants. In contrast, there were 18 garages/motor factors en-route, only 12 of these were mentioned by participants, with a total of 25 references to this landmark category.

Assuming that each participant in either the video or cognitive map group $(n=16$ in each group) could have referred to each potential landmark once, this gave a maximum possible frequency count of $32 \times$ [no. of that category en-route]. Figure 2 
Table 1. The frequency of use, and availability of, the most common landmark categories.

\begin{tabular}{lccc}
\hline $\begin{array}{l}\text { Landmark } \\
\text { category }\end{array}$ & $\begin{array}{c}\text { Total references to } \\
\text { that category } \\
\text { (all 32 participants) }\end{array}$ & $\begin{array}{c}\text { Total } \\
\text { occurrences of } \\
\text { that object en-route }\end{array}$ & $\begin{array}{c}\text { Total number of } \\
\text { those objects } \\
\text { referred to }\end{array}$ \\
\hline $\begin{array}{l}\text { Traffic lights } \\
\text { Pedestrian lights }\end{array}$ & 247 & 11 & 11 \\
Petrol stations & 77 & 7 & 7 \\
Churches & 52 & 4 & 4 \\
Sainsburys & 34 & 6 & 5 \\
Post offices & 30 & 1 & 1 \\
Bridges & 29 & 5 & 3 \\
Garages & 26 & 2 & 2 \\
Car Parks & 25 & 18 & 3 \\
Public houses & 21 & 20 & 7 \\
MacDonalds & 21 & 3 & 2 \\
\hline
\end{tabular}

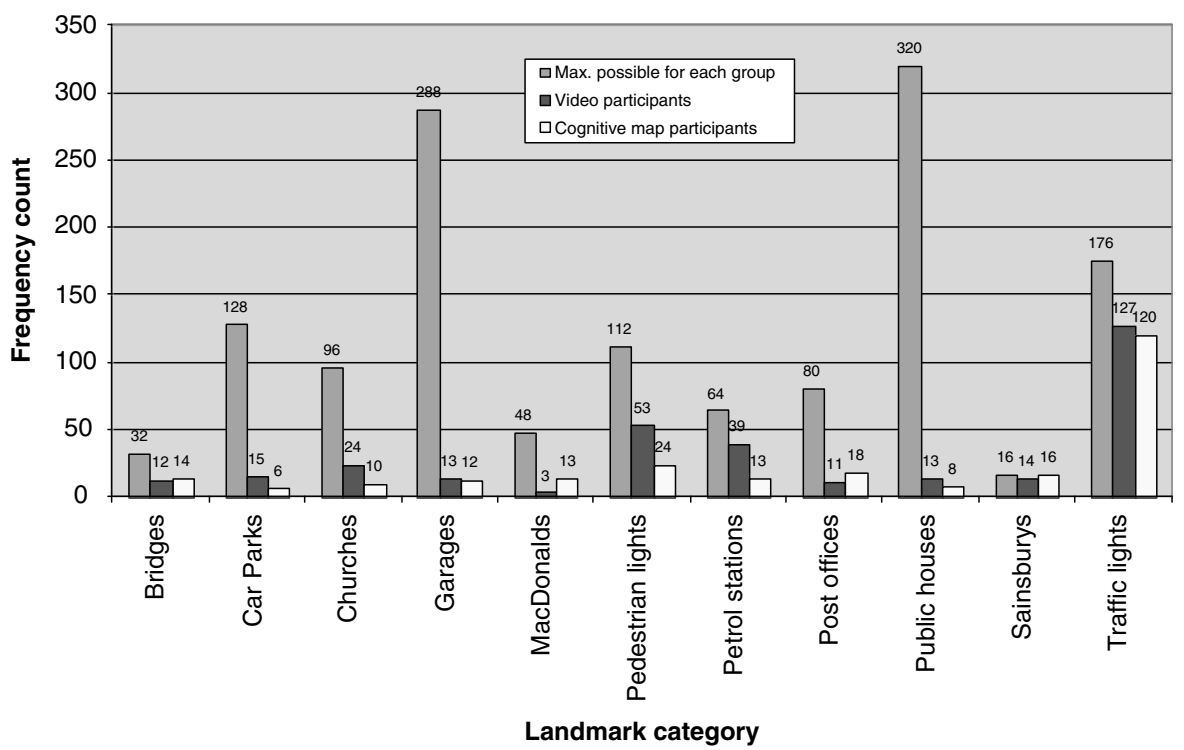

Figure 2. Actual and possible participant frequency counts for different landmark categories.

shows the frequency of, and availability of, the 11 most frequently used landmark categories.

Based on the post-trial interviews carried out with the participants, clear sets of landmark characteristics emerged that were stated as influencing their use. These were:

- Permanence: the degree to which the visual appearance or name/logo would remain constant.

- Visibility: whether the landmark can be seen from a distance and a variety of directions of travel. 
- Usefulness of location: whether a landmark is located at or near to a navigation decision point.

- Uniqueness: the likelihood of the landmark not being mistaken for other objects or features due to either being highly distinctive in appearance or not being situated near other similar looking landmarks.

- Ease of description: the ease (especially conciseness) with which the landmark can be described.

\subsection{Summary of main findings.}

- Traffic lights were the most frequently used landmark category, used widely by both the video and cognitive map participant groups.

- A prominent supermarket was used by almost all participants, as it was instantly recognisable, highly visually prominent and located near a key manoeuvre.

- Pedestrian lights and bridges were also widely used by both sets of participants.

- Landmarks such as churches were identified by the 'video' group but less so by the cognitive map group; they would appear to be distinctive in appearance but less prominent within participants' cognitive maps.

- Some public houses and garages (also including motor factors) were frequently used, but there was substantial variability in the frequency of use of individual cases of these landmark types.

- When the availability of landmarks is taken into account (Figure 2), a high proportion of traffic lights were used, but a low proportion of public houses were used.

- The characteristics which appeared to subjectively influence their use by participants included: permanence, visibility, usefulness of location, uniqueness and ease of description.

\section{REQUIREMENTS STUDY: DRIVERS' USE OF NAVIGATION INFORMATION.}

6.1. Aims. The aims of this study were to supplement the findings from the empirical work described above and to identify the landmarks used within a city (rather than town) environment. However this study had two additional aims: to analyse how landmarks were used in relation to other information cues (e.g. distance, junction descriptions etc) and to identify how information was actually used by drivers to support the task of navigating.

6.2. Method. A similar methodology to that described above was used for this study, i.e. an information generation approach based on extraction of navigation cues from either a video or an individual's cognitive map. A total of 36 participants took part in this trial, with a balance of gender and age range in each of the cognitive map and video groups. Three separate routes were used, comprising city centre, urban and out of town navigation, with a total of 38 main driver decision points.

6.3. Results. Preliminary results from this study are reported in May, Ross, \& Bayer, (2003). This previous analysis included: (1) the use of different information categories (including landmarks) by participants, (2) the degree to which information categories are used as primary or secondary information sources, and (3) whether information categories are used as either (a) preparatory information during the 


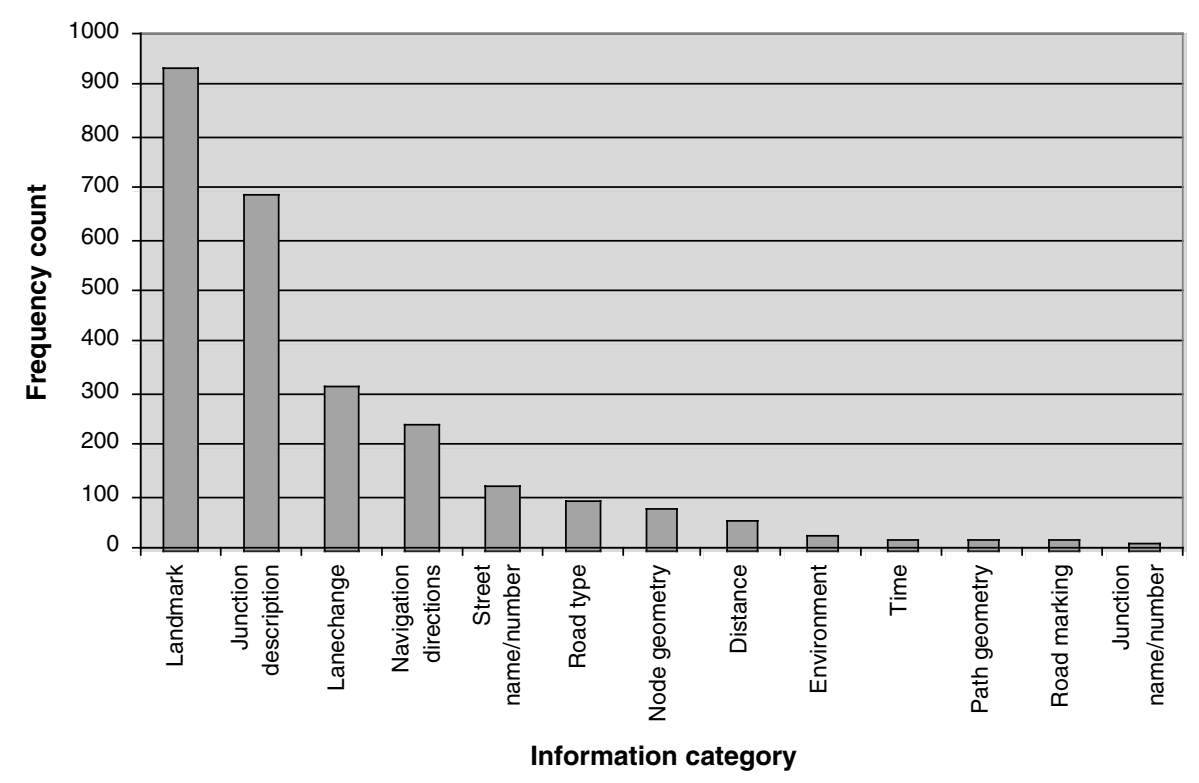

Figure 3. The frequency of use of different information cues.

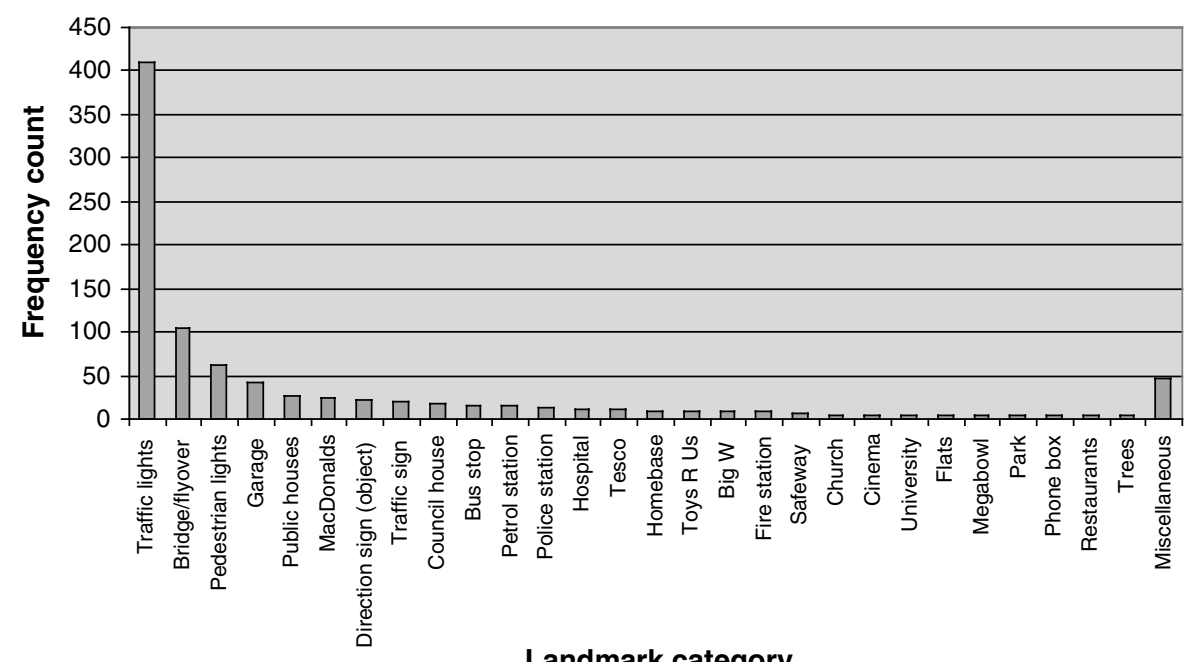

Figure 4. The frequency of use of different landmark categories.

approach to a manoeuvre (e.g. to enable appropriate speed reduction), (b) to identify the location of a turning, or (c) to provide confirmation that the correct manoeuvre has been completed. For this paper, the data was recoded to focus on the number of locations where particular information was used, rather than the total number of references to specific information cues (as a single cue may have been referred to more than once by a participant at a single location). Figure 3 shows the use of different information categories; Figure 4 gives a more detailed breakdown of the landmark 
category. Note that where individual landmarks did not readily fall into generic categories, and/or were used much more frequently than others of a similar type, they were coded individually (e.g. Fire Station, MacDonalds ${ }^{\mathrm{TM}}$ ). The miscellaneous category included all landmark categories that, when ranked in descending frequency, fell outside of a $95 \%$ cumulative frequency of use.

\subsection{Summary of main findings.}

- Landmarks were the most popular information cues generated by participants, comprising 36\% of all information cues as categorised in Figure 3. (Note that basic direction-related cues such as 'left', 'right', 'straight on' were omitted from this analysis.) The results are consistent with the survey findings of Burns, (1997) who found that, after left or right turn directions, landmarks were the second most preferred information type for identifying the location of a turn.

- A wide range of different landmarks were used (Figure 4), with over 900 references to 160 different landmarks.

- Traffic lights comprised $44 \%$ of information cues within the landmarks category, being used 4 times more often than any other landmark category.

- Distance information and street names/numbers were infrequently used by participants, in contrast to their use within many vehicle navigation systems.

Additional analysis also revealed the following regarding context of use:

- At manoeuvres, landmarks were primarily used to identify the exact location of that manoeuvre.

- Between manoeuvres landmarks were primarily used to confirm that the driver is on the correct route i.e. to build trust and confidence.

- Landmarks were infrequently used for the 'preview' stage of the navigation task.

- When landmarks were used in navigation instructions, they were usually used as the primary means of identifying a manoeuvre (as opposed to being used in addition to other information sources, i.e. as secondary or redundant information).

\section{STATISTICAL MODELLING: LANDMARK 'EFFECTIVENESS'.}

7.1. Aims \& method. To develop landmark-enhanced navigation systems for international markets, it is necessary to be able to predict which landmarks will be effective cues, based on their intrinsic qualities. The aims of this exercise were to identify the characteristics of landmarks that will predict their effectiveness as navigation cues, and determine the relative influence of each of these factors.

A range of methods was used to propose a list of characteristics that should describe the effectiveness of the landmark as a navigation cue. These included: consideration of theoretical information processing models, review of relevant applied research literature, data generated from the requirements studies outlined above, informal content analysis of video tapes of routes, analysis of participant post-trial protocols, expert human-factors assessment, and a card sort process. A set of candidate factors was then used within a stepwise linear regression analysis in order to 
Table 2. Characteristics that influence landmark effectiveness.

\begin{tabular}{|c|c|}
\hline$\overline{\mathrm{F} 1}$ & $\begin{array}{l}\text { The visual characteristics of the object and any sign or logo attached to it (ease with which you } \\
\text { can see it) }\end{array}$ \\
\hline $\mathrm{F} 2$ & The amount of required visual effort for scanning for the object (i.e. looking for it) \\
\hline F3 & The degree of pre-warning that a driver gets of the forthcoming appearance of the object \\
\hline F4 & How familiar the object is to a typical driver \\
\hline F5 & The ease of naming of the object \\
\hline F6 & $\begin{array}{l}\text { The influence of the surroundings on the ability to see the object (e.g. the existence of visual } \\
\text { clutter) }\end{array}$ \\
\hline F7 & The number of objects nearby that have a similar appearance \\
\hline F8 & $\begin{array}{l}\text { The usefulness of the location of the object for (identifying a manoeuvre/increasing driver } \\
\text { confidence) }\end{array}$ \\
\hline F9 & The level of task demands on the driver when using the landmark \\
\hline F10 & $\begin{array}{l}\text { The degree of interaction a driver normally has with the object (extent to which it is an integral } \\
\text { aspect of driving) }\end{array}$ \\
\hline
\end{tabular}

identify their potential predictive power and relative importance in terms of explaining the use of particular landmarks by participants. This analysis used the frequency of use data from the empirical studies described above, plus subjective ratings of a series of landmarks on their attributes.

7.2. Results. The factors identified as potentially influencing landmark effectiveness are shown in (Table 2) below.

Based on the regression analysis undertaken, a statistically significant model emerged $[\mathrm{F}(3,196)=35 \cdot 615, \mathrm{p}<0 \cdot 0005]$, which incorporated 3 of the factors shown in (Table 2), but accounted for only $34 \%$ of the variability in the criterion variable (landmark effectiveness). The factors that emerged with the greatest predictive power were: (1) Degree of Interaction, (2) Usefulness of Location and (3) Visual Characteristics.

\subsection{Summary of main points.}

- A set of 10 landmark factors were identified which would potentially influence the effectiveness of that landmark in a navigation context.

- The three most influential factors were shown to be:

1. The degree of Interaction that a driver has with the object (good: traffic lights as they are an integral part of the driving environment; poor: church).

2. The usefulness of location of the object (good: traffic lights as they are sited at manoeuvres and can therefore be used in relation to manoeuvres at that junction; poor: parks, as they are not associated with turnings).

3. The visual characteristics of the object and any sign or logo attached to it - the ease with which you can see it (good: petrol stations as they are large, illuminated and designed to be highly visible; poor: bus shelters).

- The predicted model was statistically significant - there was less than $0 \cdot 1 \%$ chance that the relationships established between the factors and the predicted variable were purely down to chance.

- The final model only accounted for $34 \%$ of the variability of the predicted variable (landmark effectiveness) - i.e. was only $34 \%$ effective in predicting the value of a landmark within a navigation context.

- The main reasons for unexplained variance were probably due to a number of factors, namely: the nature of the relationship between the criterion and 
predictor variables; the likelihood of other contextual influences on the use of landmarks within a real driving environment; methodological issues to do with the factor definitions, use of rating scales and the inherent variability of human judgement.

\section{ROAD TRIAL: DRIVER PERFORMANCE WITH LAND- MARKS AND DISTANCE INFORMATION.}

8.1. Aims \& method. Previous research within REGIONAL had developed an understanding of the types of landmarks (based on their defining attributes) that were 'good' or 'poor' navigation cues. The aims of this study were to assess, within a real context, driver performance when good or poor landmarks were used as key verbal information cues within navigation instructions. In addition, this study compared performance using good or poor landmarks with that achieved using verbal distanceto-turn information (instead of landmarks) to locate a manoeuvre.

A road trial was undertaken in a city in the UK (population 300,000) with a Land Rover Freelander fitted with a satellite navigation system. This system was modified to enable landmarks to be included in the verbal navigation instructions that it presented. A total of 48 participants, split into 3 balanced groups, used the satellite navigation system to navigate around a complex urban route. The navigation instructions included either 'good' landmarks, 'poor' landmarks or distance information within the auditory instructions, depending on the participant group (i.e. a between-subjects experimental design was employed). A suite of driver behavioural measures was collected during and after the trial. The following were collected in real-time during the course of the trial: visual glance data via video recording; assessment of driving errors by an accompanying qualified driving instructor, assessment of navigation errors by an accompanying experimenter, and self-reported driver confidence at three points during the approach to, and once immediately after each manoeuvre. A questionnaire was administered before, partway through, and after the trial to assess driver attitudes, and changes in attitudes, based on increasing exposure to the system. Driver workload was assessed immediately after completion of the route using a modified NASA-RTLX subjective workload scale (Fairclough, 1991; Hart \& Staveland, 1988).

Participants were pre-screened to ensure they met several key criteria: over 21, clean licence, had driven regularly for at least 3 years, had not previously used a navigation system and did not know the geographical area where the study took place. In addition, the pre-screening enabled participants to be balanced across the 3 experimental groups for gender, self-reported distance judgement ability and selfreported navigation ability.

The route that participants drove was a mixed urban/suburban route approximately $18 \mathrm{~km}$ long that took 40 minutes to drive. This route had 33 manoeuvres; analysis was based on 8 target manoeuvres where there were both good and poor landmarks that could be incorporated into the respective sets of navigation instructions presented by the satellite navigation system. Participants were unaware that there were target and non-target manoeuvres.

Detailed statistical analysis was undertaken for all measures; these details are omitted from this paper, but where experimental effects are identified below, these were all significant at $p=0.05$ or less (i.e. there was at most a $5 \%$ likelihood that the observed differences were due to chance). 


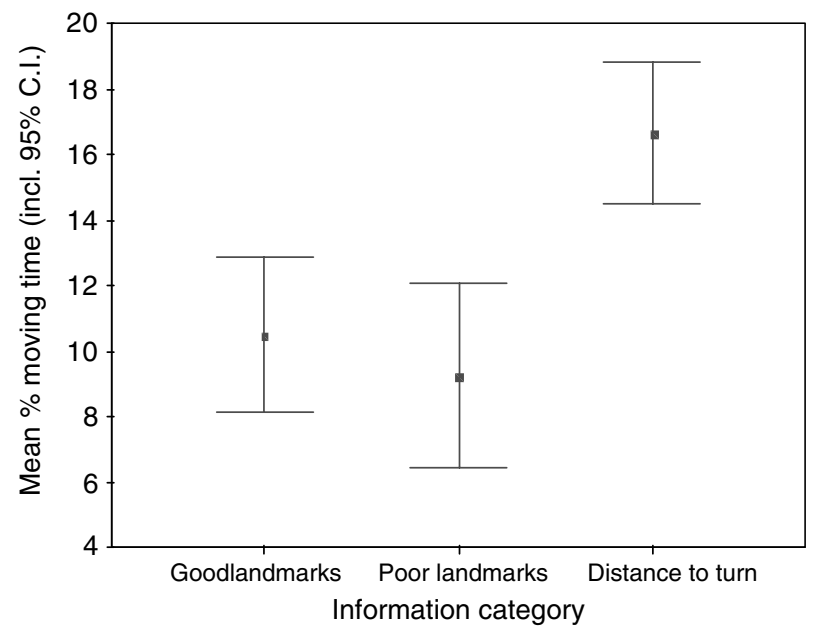

Figure 5. The effect of information category on the $\%$ moving time spent glancing to the display during the approach to a manoeuvre.

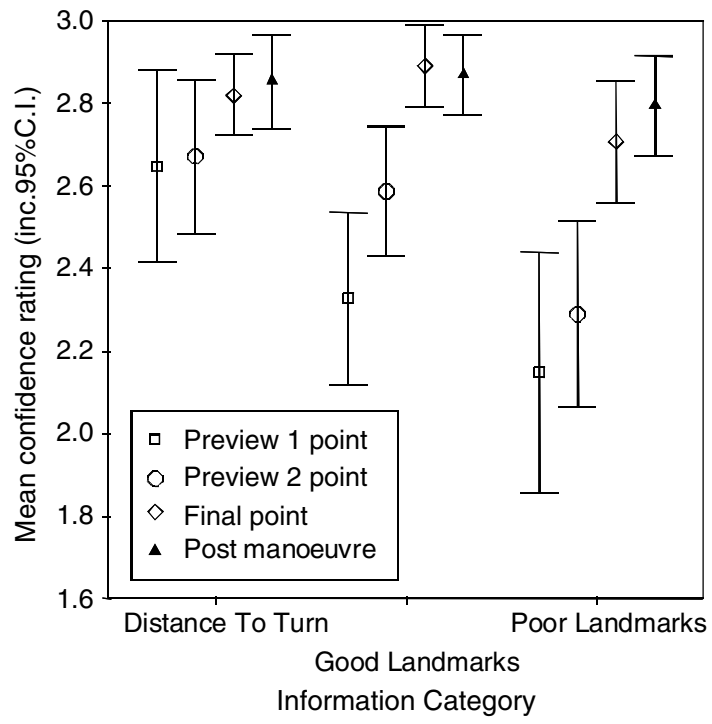

Figure 6. The effect of information category on the mean driver confidence (1: 'low'; 2 : 'medium'; 3 : 'high') at the Preview 1, Preview 2 and Final message points, and Post-manoeuvre.

8.2. Results. For the visual glance data, a percentage moving time metric was calculated based on the time spent glancing to the display as a proportion of the time spent travelling the $500 \mathrm{~m}$ approach to a manoeuvre. The means for each of the three participant groups (good landmarks, poor landmarks and distance information) are shown in Figure 5. Mean driver confidence at $450 \mathrm{~m}$ (Preview 1), $200 \mathrm{~m}$ (Preview 2), $30 \mathrm{~m}$ (Final) and immediately after each turning (Post) are shown in Figure 6 for the 3 participant groups. Note that the subjective driver confidence raw data at each of these points consisted of a rating of 'high', 'medium' or 'low', coded as 3, 2, and 1 respectively. 


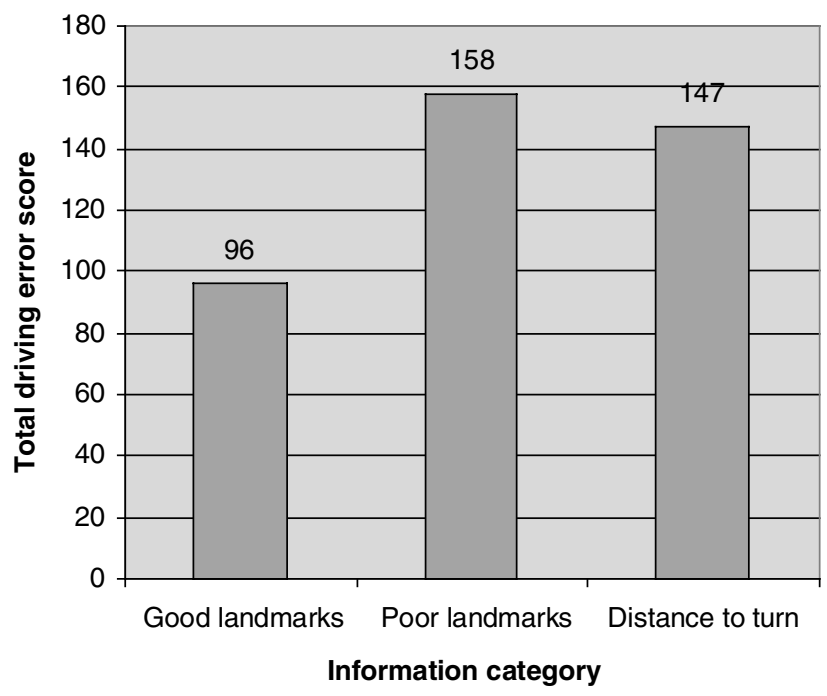

Figure 7. The effect of information category on the total driving error score per participant group.

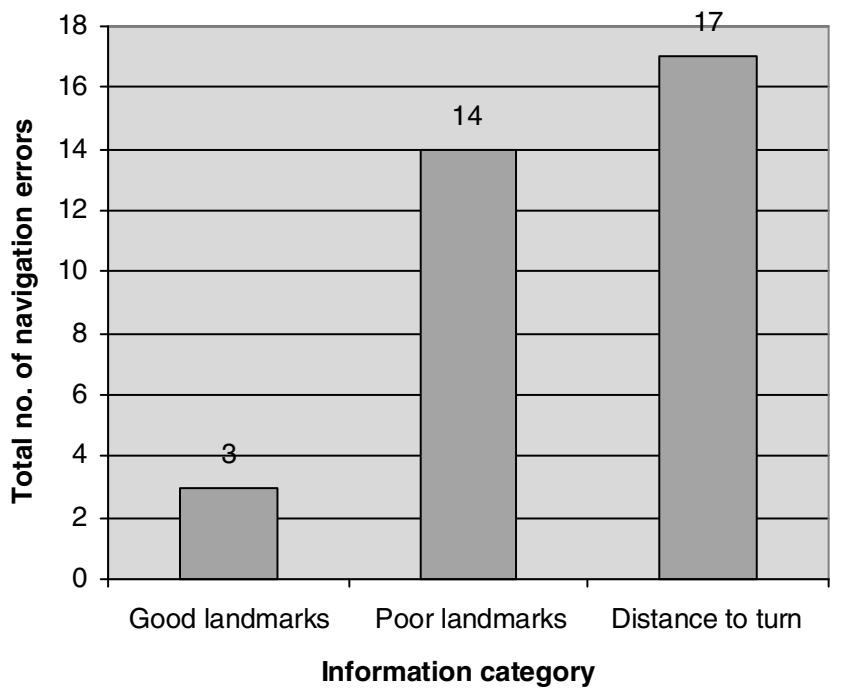

Figure 8. The effect of information category on the total of navigation errors made, per participant group.

Two main types of errors were analysed. Driving errors were coded into categories based on those used in the UK driving test, and assigned a value of 1,5 or 10 according to whether they were minor, serious or dangerous errors. The difference in the aggregated error scores between the participant groups is shown in Figure 7. Figure 8. shows the difference in the navigation errors committed by the 3 participant groups (i.e. whether good landmarks, poor landmarks, or distance information was used to locate a turning). 
The analysis of the post-trial subjective driver workload failed to demonstrate any differences between the 3 groups.

8.3. Summary of main findings.

- Incorporating landmarks within the verbal navigation instructions resulted in a $40 \%$ reduction in the percentage of approach time spent looking at the in-vehicle display. Some participants made no glances to the display during the approach to a manoeuvre when using instructions that included landmark information.

- Driver confidence at the beginning of the approach to a manoeuvre was higher when good, as opposed to poor, landmarks were used to locate turnings, but in general lower than when distance information was used instead.

- When good landmarks were used (as opposed to poor landmarks or distance information), fewer driving errors were committed by participants, consistent with the findings of Bengler et al., (1994), Philips, (1999).

- The use of good landmarks resulted in far fewer (actual or near) navigation errors than when poor landmarks or distance information were used instead.

- There were no differences in subjective driver workload between the three participant groups.

- Limited support was found for the predictive model described in Section 7: the predicted effectiveness of the landmarks used within this trial did correlate with the driver confidence levels at the manoeuvres with those landmarks. However, it was apparent that a range of other contextual factors such as traffic flow, relative size of roads, and driver's expectations about their required direction of travel also influenced the driving and navigating performance during the trial.

\section{ROAD TRIAL: NAVIGATION USING TRAFFIC LIGHTS.}

9.1. Aims. Previous research indicated that traffic lights can be an effective navigational landmark. However little research had been undertaken to determine how they might be actually used as navigation cues, and the impact that context has on their effectiveness. The aims of this study were to determine: (1) whether traffic lights could be used as navigational landmarks where several sets are sited closed together; (2) whether drivers distinguished between traffic lights and pedestrian lights; (3) the impact of terminology on driver performance; and (4) the extent to which counting strategies could be employed effectively.

9.2. Method. This study was an empirical road-based trial, where 30 participants were presented with verbal navigation instructions incorporating traffic light information. They were then asked to complete a series of manoeuvres based on these instructions. Participants received instructions that were either of the form 'turn left at the traffic lights' or 'turn left at the set of traffic lights'. Navigation errors and strategies were captured for each manoeuvre. Two complete circuits of an inner city/ urban course were completed. On the first circuit, navigational errors and driver confidence were determined, on the second circuit of the same route, counting strategies were investigated. The test route consisted of three straight sections of an approximately triangular route. Each of these three parts of the route contained a series of traffic lights and pedestrian lights, and within the constraints of a real road environment, represented a 'high', 'medium' and 'low' density of lights. 


\subsection{Summary of main findings:}

- Where manoeuvres were at junctions controlled by traffic lights, participants were able to easily identify the required turning, even when the density of traffic lights/pedestrian lights along a stretch of road was high. Where a manoeuvre was adjacent to a set of pedestrian lights (as opposed to located at traffic lights), drivers were less successful, with an overall error rate of up to $50 \%$.

- Participants generally did not differentiate between pedestrian lights and traffic lights, i.e. when presented with an instruction of the form 'turn right at the second set of traffic lights' they generally included any occurrences of pedestrian lights within their counting strategy.

- Navigation instructions that referred to 'sets of traffic lights' rather than 'traffic lights' resulted in fewer navigation errors, although this effect was only readily apparent for the most complex navigation task where the greatest navigational uncertainty existed.

- Based on the results of a dynamic counting task, participants were able to apply a counting strategy successfully for up to four occurrences of traffic lights. However this result does not take into account additional navigating demands, which are likely to reduce the performance on this kind of task.

\section{ROAD TRIAL (3): IMPACT OF INFORMATION RELIABILITY.}

10.1. Aims. If landmarks are incorporated in either onboard or offboard navigation systems, this necessitates information on them being held on a navigable database. Keeping this information up to date is an intrinsic problem that database manufacturers have to overcome. Some categories of landmark will experience more frequent change than others (e.g. the names of public houses change frequently, church names rarely do). One solution to name changes is to only use generic terms, e.g. 'public house', 'petrol station' but this would reduce the potential benefits of landmarks and would be seen by industry as a retrograde step (fuel brand logos are already used to identify petrol stations on digital maps). Little research exists which investigates the impact on the driver of unreliable navigation information. The aim of this study was to assess the impact on driver confidence and navigation performance if the landmarks provided as navigation cues were incorrect.

10.2. Method. Eighteen participants (all over 21, with a clean license and split $50: 50$ for gender) drove three routes, each route being devised such that one category of landmark could be used within the navigation instructions used to navigate that route successfully. Navigation instructions for each route incorporated either traffic lights at 10 manoeuvres, public houses at 10 manoeuvres or petrol stations at 10 manoeuvres. All 'target' manoeuvres (i.e. those using landmarks) were linked by several other manoeuvres without landmarks to ensure a continuous route. For the route utilising traffic lights as landmarks, there were no errors in the information given. Where public houses and petrol stations were used, the 7th occurrence of the landmark was correctly identified as either a public house or petrol station, but was incorrectly named.

Participants navigated the three routes using simulated verbal navigation instructions generated using a PC. In most cases, three instructions were given for each 


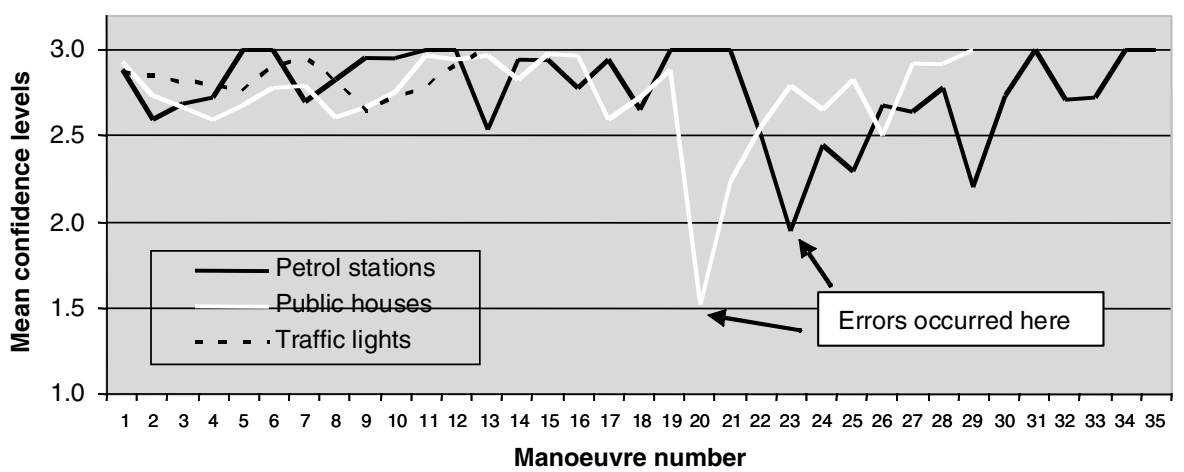

Figure 9. Effect of naming errors on drivers' confidence levels.

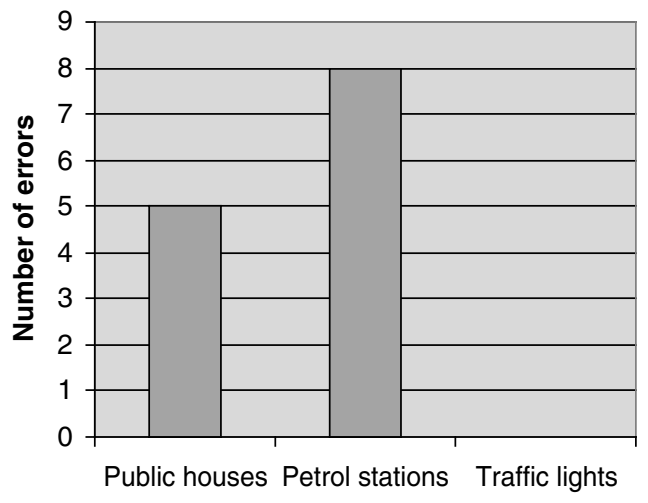

Type of landmark

Figure 10. The number of navigation errors committed with landmark types.

manoeuvre, at distances of approximately 500, 200 and $50 \mathrm{~m}$ from the turning (reflecting the operation of many current vehicle navigation systems). A typical instruction was 'in $500 \mathrm{~m}$ turn right after the BP petrol station'. After each instruction, participants were required to give a confidence rating of 'high', 'medium' or 'low' to indicate how confident they were that they would be able to identify the correct turn and complete the manoeuvre successfully. The completion order of the routes was counterbalanced within the repeated measures experimental design. Driver attitudinal data was collected after the completion of each route, and at the end of the trial.

10.3. Results. Figure 9 shows the variation in driver confidence over the course of the trial. Note that confidence ratings of 'high', 'medium' and 'low' were coded as 3,2 and 1 respectively.

Figure 10 and Figure 11 show the number of navigation errors and preferences for particular landmark categories.

10.4. Summary of main findings.

- A 3 to $4 \%$ navigational error rate occurred at manoeuvres supported by public houses or petrol stations. 


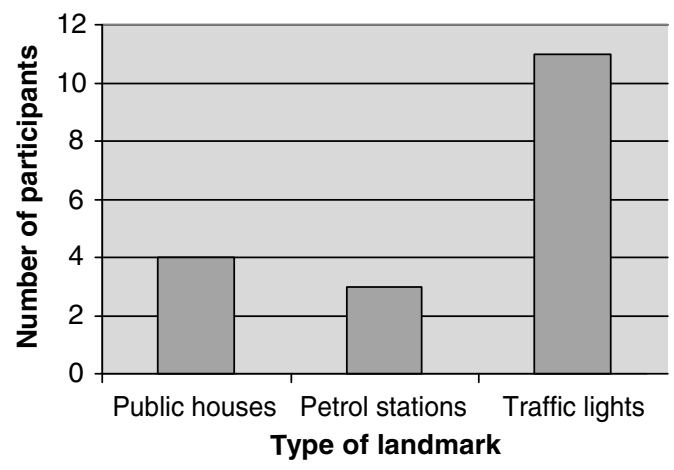

Figure 11. Participants' preferred landmark for navigating.

- Traffic lights were preferred as a navigational landmark by over $60 \%$ of the participants.

- Only half $(n=9)$ of the participants actually noticed the error in naming of the petrol station; most $(n=16)$ noticed the error in the naming of the public house. Since the basic form of a petrol station is highly visible from a distance and they are relatively infrequent, participants probably assumed that the one visible was that being referred to, especially as no logo was presented.

- The pre-error confidence range was $2 \cdot 5-3 \cdot 0$. For the manoeuvre at which the error occurred, mean confidence dropped to $2 \cdot 0$ for petrol stations and 1.5 for pubs (both significant falls in confidence). The falls in confidence are likely to be directly related to the degree to which the landmark was identified as incorrect, and hence the perceived navigational uncertainty.

- Prior to the error occurring, all categories of landmark induced a similar level of mean driver confidence (traffic lights $=2.83$; pubs $=2.79$; petrol stations $=$ $2 \cdot 85)$. Post-error, mean driver confidence dropped to 2.63 for petrol stations and $2 \cdot 29$ for pubs.

- After the error occurred, mean confidence did not return to pre-error levels until 3 manoeuvres later.

11. REGIONAL PROJECT-CONCLUSIONS. The aim of the REGIONAL project was to carry out new research to enable landmarks to be an integral feature of future vehicle navigation systems. A range of theoretical and empirical studies was undertaken out to determine: the types of landmarks that are effective navigation cues and factors which influence this; their impact on driver performance; the effect of data reliability, and the use of traffic lights as navigation landmarks.

Landmarks have the potential to make navigation systems more effective and easier to use than where distance and road layout alone are used to locate forthcoming manoeuvres for a driver. In particular, landmarks were shown to decrease the reliance on a visual display, increase driver confidence and satisfaction, decrease driver navigation errors, and positively impact on driver safety.

Some categories of landmarks, particularly traffic lights, are consistently effective navigation cues. However other landmark categories, including some which are 
traditionally seen as 'good' navigation cues (e.g. public houses, churches) are less effective, with great variation in the effectiveness of individual objects within these categories. Although good landmarks are potentially highly beneficial to a driver, the use of poor landmarks (e.g. those difficult to see or distinguish from others, poorly sited, or difficult to describe) can actually be detrimental within a driving and navigating context.

There are identifiable factors which clearly influence the effectiveness of landmarks within a real driving environment. In addition, the context of a manoeuvre has a major influence on a driver's requirement for navigation information and hence the potential added value that a landmark may provide. Navigation systems need to integrate information on relative road sizes, signage, prior/subsequent junctions, lane positioning, as well as landmarks in order to provide the key context-relevant information that a driver needs to navigate effectively and safely.

There are undoubtedly potential customer benefits in incorporating landmarks in vehicle navigation systems. However it is likely that the business case will determine the pace with which landmarks are actually employed in next generation systems.

\section{ACKNOWLEDGEMENTS}

The REGIONAL project was funded by the UK government under the LINK Inland Surface Transport Programme (EPSRC). The authors are employed at ESRI, Loughborough University, and gratefully acknowledge the support of the other project partners: Jaguar Cars Ltd, Alpine Electronics UK Ltd, Navigation Technologies (NAVTEQ), Motor Industry Research Association (MIRA) and RAC Motoring Services. The views expressed in this paper are solely those of the authors.

\section{REFERENCES}

Alm, H. (1990). Drivers' cognitive models of routes. In J. A. Rothengatter (Ed.), Laboratory and field studies on route representation and drivers' cognitive models of routes (DRIVE II V1041 GIDS, Deliverable GIDS/NAV2) (pp. 35-48). Groningen, The Netherlands: University of Groningen, Traffic Research Centre.

Alm, H., Nilsson, L., Jarmark, S., Savelid, J., \& Hennings, U. (1992). The effects of landmark presentation on driver performance and uncertainty in a navigation task - a field study (No. S/IT-4): (Swedish Prometheus, Tech. Rep. No. S/IT-4). Linköping, Sweden: VTI.

Bengler, K., Haller, R., \& Zimmer, A. (1994). Experimental optimisation of route guidance information using context information. Paper presented at the First World Congress on Applications of Transport and Intelligent Vehicle Highway Systemsf, Paris, France.

Burnett, G. E. (1998). "Turn right at the King's Head": Drivers' requirements for route guidance information. Unpublished PhD thesis, Loughborough University, UK.

Burns, P. (1997). Navigation and the older driver. Unpublished PhD thesis, Loughborough University, Loughborough, UK.

Evans, G. W., Skorpanich, A. A., Garling, T., Bryant, K. J., Bresolin, B. (1984). The effects of pathway configuration, landmarks and stress on environmental cognition. Journal of environmental psychology, 4(4), 323-335.

Fairclough, S. H. (1991). Adapting the TLX to Measure Driver Mental Workload (DRIVE Project V1017 (BERTIE) No. Report no. 71). Loughborough, UK: HUSAT Research Institute.

Golledge, R. G. (1993). Geographical perspectives on spatial cognition. In T. Garling, Golledge, R. G. (Ed.), Behaviour and Environment: psychological and geographical approaches (pp. 16-46). Amsterdam: Elsevier Science Publishers B. V.

Green, P., Hoekstra, E., Williams, M., Wen, C., \& George, K. (1993). Examination of a videotapebased method to evaluate the usability of route guidance and traffic information systems (Final 
No. UMTRI-93-31): (Tech. Rep. No. UMTRI-93-31). Ann Arbor, MI: University of Michigan Transportation Research Institute.

Hart, S. G., \& Staveland, L. E. (1988). Development of NASA-TLX (Task Load Index): results of empirical and theoretical research. In N. Meshkati (Ed.), Human Mental Workload (pp. 139-183). Amsterdam: North-Holland.

King, G. F. (1986). Driver performance in highway navigation tasks. Transportation research record(1093), 1-11.

May, A. J., Ross, T., \& Bayer, S. H. (2003). Drivers' Information Requirements when Navigating in an Urban Environment. The Journal of Navigation, 56, 89-100.

Pauzie, A., Daimon, T., Bruyas, M. (1997). How to design landmarks for guidance systems? Paper presented at the 4th World Congress on ITS.

Philips, B. H. (1999, September 27-October 1, 1999). The Role of Landmark Information in Intelligent Navigation Displays. Paper presented at the Houston... We Have a Solution!, Proceedings of the Human Factors and Ergonomics Society 43rd Annual Meeting.

Streeter, L. A., Vitello, D. (1986). A profile of drivers' map-reading abilities. Human factors, 28(2), 223-239.

Wierwille, W. W. (1993). Visual and manual demands of in-car controls and displays. In B. Peacock, Karwowski, W. (Ed.), Automotive Ergonomics (pp. 299-320). London: Taylor and Francis.

Wochinger, K., \& Boehm-Davis, D. (1997). Navigational preference and driver acceptance of advanced traveler information systems. In I. Y. Noy (Ed.), Ergonomics and safety of intelligent driver interfaces (pp. 345-362). Mahwah, NJ: Lawrence Erlbaum Associates. 